\title{
Impairment of Immunonutritional Status During Treatment is a Factor Associated With Oncologic Outcomes in Patients With Rectal Cancer Treated With Preoperative Chemoradiotherapy
}

\author{
In Ja Park \\ Department of Colon and Rectal Surgery, Asan Medical Center, University of Ulsan College of Medicine, Seoul, Korea
}

See Article on Page 208-214

Studies regarding preoperative nutritional and immunologic status have increased. The prognostic nutritional index (PNI) calculated based on the serum albumin concentration and the total lymphocyte count was initially suggested for assessing the influence of the perioperative immunonutritional status on surgical risk [1]. Recently, studies have reported an association of the PNI with oncologic outcomes and with postoperative complications in patients with various types of cancers [2-4]. These studies reflect the increasing interest in correcting environmental factors to improve oncologic outcomes.

Although preoperative chemoradiotherapy (PCRT) has been established as one of the standard treatments for patients with locally advanced rectal cancer, many suggestions have been made and many controversies exist regarding prognostic factors. The first step in identifying prognostic factors has to be an evaluation of known prognostic factors for patients who were not treated with PCRT.

In colorectal cancer patients, malnutrition is known to be related with the risk of postoperative complications, and postoperative complications induce chronic inflammation, which might lead to worse long-term oncologic outcomes [5]. However, the mechanism of oncologic impairment due to malnutrition has not been

Correspondence to: In Ja Park, M.D.

Department of Colon and Rectal Surgery, University of College of Medicine and Asan Medical Center, 88 Olympic-ro 43-gil, Songpa-gu, Seoul 05505, Korea

Tel: +82-2-3010-3937, Fax: +82-2-474-9027

E-mail: ipark@amc.seoul.kr

(C) 2016 The Korean Society of Coloproctology

This is an open-access article distributed under the terms of the Creative Commons Attribution NonCommercial License (http://creativecommons.org/licenses/by-nc/4.0) which permits unrestricted noncommercial use, distribution, and reproduction in any medium, provided the original work is properly cited. clearly demonstrated. The authors of "Prognostic impact of immunonutritional status changes during preoperative chemoradiation in patients with rectal cancer" evaluated the influence of the PNI in the setting of PCRT [6] by using the difference between the preCRT PNI and the post-CRT PNI (dPNI). When impairment of the immunonutritional status during PCRT was low, oncologic outcomes were better, even when other potent prognostic factors were considered. Based on their results, patients' long-term outcomes may be enhanced by correcting their immunonutritional status during PCRT.

Before accepting the results of this study, we need to investigate the influence of initial immunonutritional status on oncologic outcome. A PNI of 45 is defined as moderate to severe malnutrition. If the cutoff value of malnutrition is considered, the cutoff vale of 5 for the dPNI seems small and may not sufficiently reflect the nutritional status. Therefore, the possibly that the association of the dPNI with oncologic outcome was influenced by the pretreatment nutritional status needs to be tested. Once this is accomplished, one can proceed to investigating the effect that correcting the nutritional status would have on long-term oncologic outcomes.

The immunonutritional status of patients should be one of the main points of interest in treating patients with colorectal cancer. This is important because the immunonutritional status of the patient is a modifiable factor. For this reason, we expect studies regarding immunologic or nutritional status in patients with colorectal cancer to increase over time. However, we need to be careful in analyzing the results of such studies to find proper applications in clinical practice.

\section{CONFLICT OF INTEREST}

No potential conflict of interest relevant to this article was reported. 


\section{REFERENCES}

1. Onodera T, Goseki N, Kosaki G. Prognostic nutritional index in gastrointestinal surgery of malnourished cancer patients. Nihon Geka Gakkai Zasshi 1984;85:1001-5.

2. Schwegler I, von Holzen A, Gutzwiller JP, Schlumpf R, Mühlebach S, Stanga Z. Nutritional risk is a clinical predictor of postoperative mortality and morbidity in surgery for colorectal cancer. Br J Surg 2010;97:92-7.

3. Migita K, Takayama T, Saeki K, Matsumoto S, Wakatsuki K, Enomoto $\mathrm{K}$, et al. The prognostic nutritional index predicts longterm outcomes of gastric cancer patients independent of tumor stage. Ann Surg Oncol 2013;20:2647-54.

4. Mori S, Usami N, Fukumoto K, Mizuno T, Kuroda H, Sakakura N, et al. The significance of the prognostic nutritional index in patients with completely resected non-small cell lung cancer. PLoS One 2015;10:e0136897.

5. Nozoe T, Kohno M, Iguchi T, Mori E, Maeda T, Matsukuma A, et al. The prognostic nutritional index can be a prognostic indicator in colorectal carcinoma. Surg Today 2012;42:532-5.

6. Lee YJ, Kim WR, Han J, Han YD, Cho MS, Hur H, et al. Prognostic impact of immunonutritional status changes during preoperative chemoradiation in patients with rectal cancer. Ann Coloproctol 2016;32:208-14. 